\title{
Stage III Mycosis Fungoides AJCC v7
}

National Cancer Institute

\section{Source}

National Cancer Institute. Stage III Mycosis Fungoides A/CC v7. NCI Thesaurus. Code C7800

Stage III includes: (T4, N0-2, M0, B0-1); IIIA (T4, N0-2, M0, B0); IIIB (T4, N0-2, M0, B1).

T4: Confluence of erythema covering $80 \%$ or more of body surface area. N0: No clinically abnormal peripheral lymph nodes; biopsy not required. N1: Clinically abnormal peripheral lymph nodes; histopathology Dutch grade 1 or NCI LN0-2. N2: Clinically abnormal peripheral lymph nodes; histopathology Dutch grade 2 or NCI LN3. MO: No visceral org an involvement. B0: Absence of significant blood involvement: $5 \%$ or less of peripheral blood lymphocytes are atypical (Sezary cells). B1: Low blood tumor burden: more than $5 \%$ of peripheral blood lymphocytes are atypical (Sezary cells) but does not meet the criteria of B2. (AJCC 7th ed.) 